\title{
Clinical efficacy and safety of enhanced recovery after surgery for patients treated with radical cystectomy and ileal urinary diversion: a systematic review and meta-analysis of randomized controlled trials
}

\author{
Dechao Feng", Shengzhuo Liu", Yiping Lu, Wuran Wei, Ping Han \\ Department of Urology, Institute of Urology, West China Hospital, Sichuan University, Chengdu, China \\ Contributions: (I) Conception and design: D Feng; (II) Administrative support: P Han, Y Lu, W Wei; (III) Provision of study materials or patients: \\ D Feng, S Liu, Y Lu; (IV) Collection and assembly of data: D Feng, S Liu; (V) Data analysis and interpretation: D Feng, S Liu; (VI) Manuscript \\ writing: All authors; (VII) Final approval of manuscript: All authors. \\ "These authors contributed equally to this work. \\ Correspondence to: Wuran Wei; Ping Han. Department of Urology, Institute of Urology, West China Hospital, Sichuan University, Guoxue Xiang \\ \#37, Chengdu 610041, Sichuan, China. Email: weiwuranwch@126.com; hanpingwch@163.com.
}

\begin{abstract}
The aim of this study was to evaluate the effect of enhanced recovery after surgery (ERAS) on perioperative outcomes in patients undergoing radical cystectomy (RC) and ileal urinary diversion (IUD). We performed a literature search of PubMed, Web of Science, EMBASE, the Cochrane Library and three main Chinese databases (WANFANG, CNKI and VIP) in December 2019 without language restrictions. Two reviewers independently selected studies, evaluated methodological quality and extracted data using Cochrane Collaboration's tools. Efficacy was assessed by the time to first flatus, first bowel movement, and hospitalization time. Safety was assessed by 30-day readmission and complications after surgery. Our searches identified 6 studies, including 628 patients. A total of $323(51 \%)$ patients took ERAS. We observed that ERAS reduced the time to first flatus [standard mean difference (SMD): $-1.65,95 \% \mathrm{CI}:-2.63$ to -0.68 , $\mathrm{P}=0.0009]$, first bowel movement (SMD: -1.14 , 95\% CI: -1.78 to $-0.50, \mathrm{P}=0.0005$ ), and hospitalization time (MD: $-4.09,95 \% \mathrm{CI}:-6.34$ to $-1.85, \mathrm{P}=0.0004)$. We did not detect significant difference in terms of 30-day readmission [relative risk (RR): 1.33, 95\% CI: 0.61-2.88, $\mathrm{P}=0.48$ ] and postoperative complications (RR: $0.91,95 \%$ CI: $0.65-1.26, \mathrm{P}=0.56$ ) between ERAS and conventional recovery after surgery (CRAS). Our findings indicated that ERAS protocols throughout the perioperative period of RC with IUD might reduce hospitalization expenses and contribute to higher turnover ward, more efficient utilization of medical resources and lower risk of nosocomial infection as a result of shorter length of stay. Besides, early rehabilitation of gastrointestinal function might not only facilitate wound healing and early mobilization, thereby reducing the incidence of basic complications such as cardiopulmonary disease, but also improve patients' psychological trauma and stress response, increase self-confidence and motivation in treatments, and then lead to unexpected benefits. Further large volume, multicenter randomized controlled studies are warranted before making the final clinical guidelines.
\end{abstract}

Keywords: Enhanced recovery after surgery (ERAS); radical cystectomy (RC); bladder cancer; randomized controlled trials (RCTs)

Submitted Dec 31, 2019. Accepted for publication Jun 09, 2020.

doi: 10.21037/tau-19-941

View this article at: http://dx.doi.org/10.21037/tau-19-941 


\section{Introduction}

Bladder cancer is one of the most common fatal urological malignancies worldwide (1), which has troubled patients a lot and brought serious economic burden to many families. Radical cystectomy (RC) with urinary diversion (UD) has been currently the gold standard for patients with muscle invasive bladder cancer and high-risk non-muscle invasive bladder cancer (2). RC with UD is a complex surgical procedure involving urinary system and intestinal system, which is associated with considerable perioperative mortality and morbidity (3).

Enhanced recovery after surgery (ERAS) or multidisciplinary fast-track surgery was initially described by Kehlet (4) in the late 1990s, which aimed to minimize surgical trauma and stress response, reduce surgical related complications, and accelerate postoperative recovery in the perioperative period. The concept of ERAS has been widely used in various operations in the past decades, especially in colorectal surgery (5). In recent years, ERAS has gradually attracted the attention of urologists with the development of minimally invasive $\mathrm{RC}$, the focus on the concept of organ function protection and the improvement of UD technology. The previous meta-analysis (6-9) performed cumulative analyses of a large number of published observational studies, which favored the efficacy of ERAS for the perioperative management of RC with UD. However, the inherent limitations of observational studies biased the pooled estimates in favor of ERAS, thus lowering the level of evidence of the previous meta-analysis (6-9). Herein, we conducted a systematic review and metaanalysis comparing ERAS to conventional recovery after surgery (CRAS) to provide a higher level of evidence and guide clinical practice based on randomized controlled trials (RCTs) at home and abroad. We present the following article in accordance with the PRISMA reporting checklist (available at http://dx.doi.org/10.21037/tau-19-941).

\section{Methods}

\section{Study selection}

In accordance with the PRISMA guidelines (10), a systematic literature search was conducted in December 2019 based on computerized databases including PubMed, Web of Science, EMBASE, the Cochrane Library and three main Chinese databases (WANFANG, CNKI and VIP) without language restrictions. Search terms or keywords used included bladder cancer, enhanced recovery after surgery, radical cystectomy, and fast track surgery. The search strategy used in PubMed was as follows: Search ((( Fast track surgery [Title/Abstract]) OR enhanced recovery after surgery protocols [Title/Abstract]) OR ERAS[Title/Abstract])) AND radical cystectomy [Title/ Abstract]. Moreover, we performed a manual search of the reference lists of the included studies or relevant review to ensure literature saturation. All RCTs reporting the outcomes interested were included.

\section{Selection criteria}

The eligibility of studies was defined using the PICOS method: Patients $(\mathrm{P})$ : patients treated with $\mathrm{RC}$ and ileal urinary diversion (IUD) through open, laparoscopic or robot-assisted approach; Intervention (I): ERAS; Comparison (C): publications comparing ERAS to CRAS; Outcomes $(\mathrm{O})$ : efficacy was assessed by the time to first flatus, first bowel movement, and hospitalization time. Safety was assessed by 30 -day readmission and postoperative complications. The complications included ileus, deep vein thrombosis, infection of incision, intestinal fistula, leakage of urine, urinary tract infection and wound healing disorders. Study design (S): RCTs. Studies with overlapping or insufficient data were excluded. We also excluded review, lectures, meeting abstracts and meta-analysis. Three independent reviewers (D Feng, S Liu and Y Lu) screened study based on titles and abstracts. Studies that satisfied the inclusion criteria were retrieved for full-text assessment. Data were independently extracted by two reviewers (D Feng, S Liu). Discrepancies were resolved by another researchers (W Wei). The manuscript was revised by the reviewer (P Han).

\section{Quality assessment}

Two independent authors (D Feng, S Liu) evaluated the methodological quality of the studies (Figure 1) according to the Cochrane Collaboration's Risk of Bias (RoB) tool in Review Manager software (https://community.cochrane. org/help/tools-and-software/revman-5). This tool primarily evaluates 7 domains: random sequence generation (selection bias); allocation concealment (selection bias); blinding of participants and personnel (performance bias); blinding of outcome assessment (detection bias); incomplete outcome data (attrition bias); selective reporting (reporting bias); other bias (such as funding sources).

D Feng and S Liu independently rated the level of evidence of the included articles using the Oxford Centre 

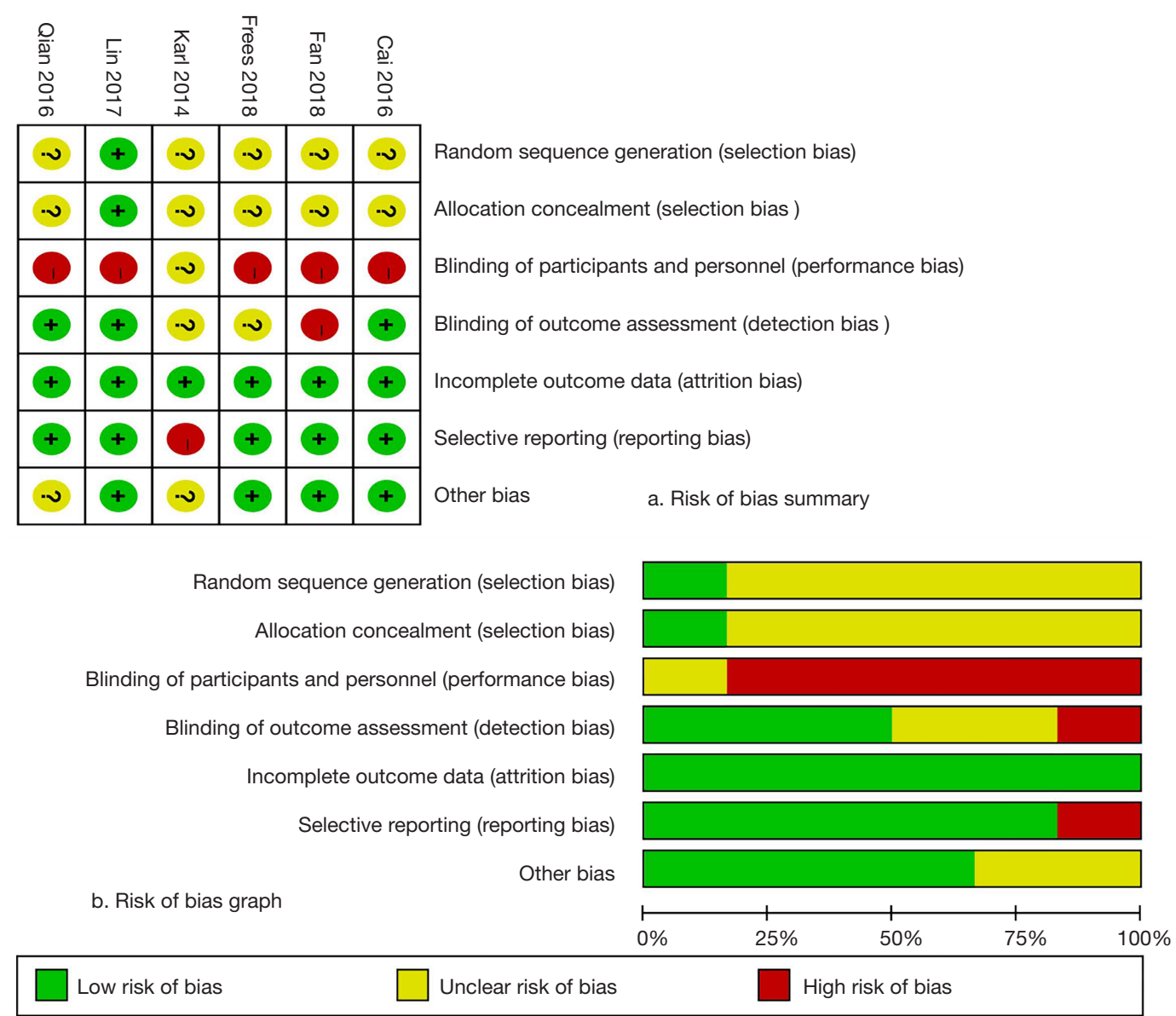

Figure 1 The risk of bias summary of included studies.

for Evidence-Based Medicine criteria (11). This scale graded studies from strongest (level 1) to weakest (level 5) strength of evidence according to study design and data quality.

Figure 1 presents the RoB summary of the 6 RCTs (12-17). In general, included studies had a low risk of attrition bias (incomplete outcome data) and reporting bias (selective reporting). However, the risk of performance biases was high. The risk of selection bias was unclear due to lack of related description.

\section{Statistical analysis}

The continuous and dichotomous variables were described as means \pm standard deviation (SD) and proportions, respectively. Median and range were used to estimate mean and SD (18). The percentiles, 25 th and 75 th percentiles as well as 5 th and 95 th percentiles, were transformed to SD through the following formula: $\mathrm{SD} \approx$ Norm IQR $=$ $(\mathrm{P} 75-\mathrm{P} 25) \times 0.7413$ (IQR: inter-quartile range, P75: 75th percentile, P25: 25th percentile) (19).

We calculated pooled estimates of the mean difference (MD) or standard mean difference (SMD) and relative risk (RR) for continuous and dichotomous variables, respectively. We used the Cochran $\mathrm{Q}$ test to assess betweenstudy heterogeneity (20). We also did $\mathrm{I}^{2}$ testing to evaluate the magnitude of the heterogeneity with values $>50 \%$ regarded as being significant heterogeneity (21). The random effects model was used when the trials yielded heterogeneity $(\mathrm{P}<0.1)$. Otherwise, the fixed effects model was used. Significance was set at $\mathrm{P}<0.05$. We conducted a subgroup analysis according to the type of UD. This metaanalysis was accomplished by RevMan5 (version 5.3).

\section{Results}

\section{Search results}

Four hundred possible studies were identified from the 


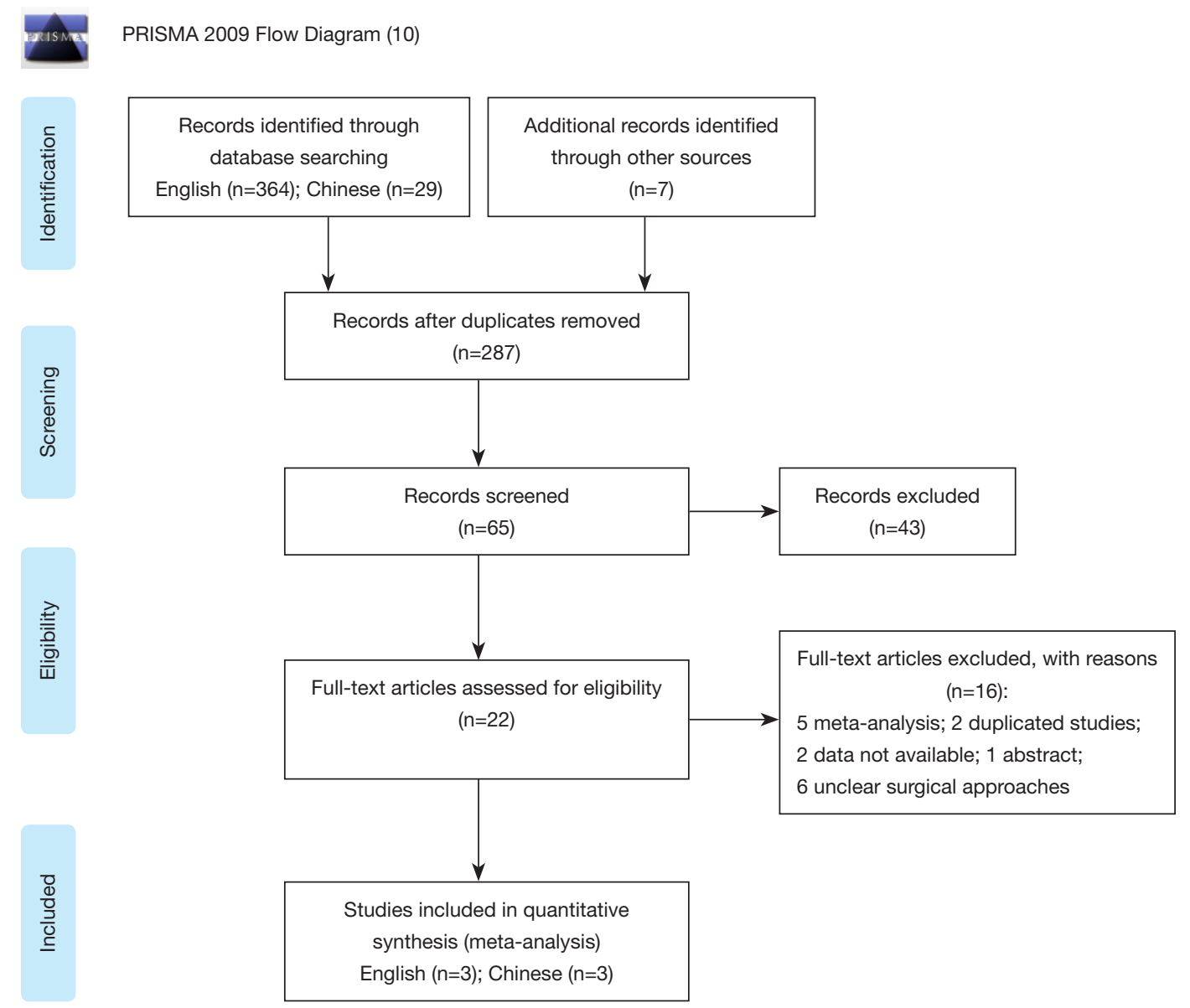

Figure 2 The study selection process (10).

database and 6 trials (12-17) comparing ERAS to CRAS were included in the final analysis with 3 English articles (12-14) and 3 Chinese articles (15-17). The study selection process is summarized in Figure 2, and Table 1 details the characteristics of enrolled studies.

\section{Clinical efficacy of ERAS}

Five studies (13-17) reported the time to first flatus with 527 consecutive patients undergoing ERAS $(\mathrm{n}=261)$ and CRAS $(n=266)$. The pooled analysis showed a significantly shorter time to flatus in the ERAS group compared with control group (SMD: $-1.65,95 \% \mathrm{CI}:-2.63$ to $-0.68, \mathrm{P}=0.0009$; Figure 3). There was statistical heterogeneity between the trials $\left(\mathrm{P}<0.00001 ; \mathrm{I}^{2}=94 \%\right)$.

Four studies $(13,14,16,17)$ containing 487 patients $(241$ in the ERAS group and 246 in the CRAS group) reported the time to first bowel movement. The pooled analysis showed a significantly shorter time to first bowel movement in the ERAS group compared to CRAS group (SMD: -1.14, 95\% CI: -1.78 to $-0.50, \mathrm{P}=0.0005$; Figure 3) with highly between-study heterogeneity $\left(\mathrm{P}<0.0001, \mathrm{I}^{2}=87 \%\right)$.

Five studies (13-17) contained 527 patients reported the result of hospitalization time. Patients in the ERAS group experienced shorter hospitalization time compared to CRAS group (MD: $-4.09,95 \% \mathrm{CI}:-6.34$ to $-1.85, \mathrm{P}=0.0004$; Figure 3). There was statistical heterogeneity between the trials $\left(\mathrm{P}<0.00001 ; \mathrm{I}^{2}=96 \%\right)$.

\section{Clinical safety of ERAS}

Three studies $(13,14,16)$ contained 427 patients $(211$ in the ERAS group and 216 in the CRAS group) reporting 30 -day readmission. The pooled data from the randomeffects model demonstrated no significant difference between the ERAS and SC groups (RR: 1.33, 95\% CI: 
Table 1 The baseline characteristics of included studies

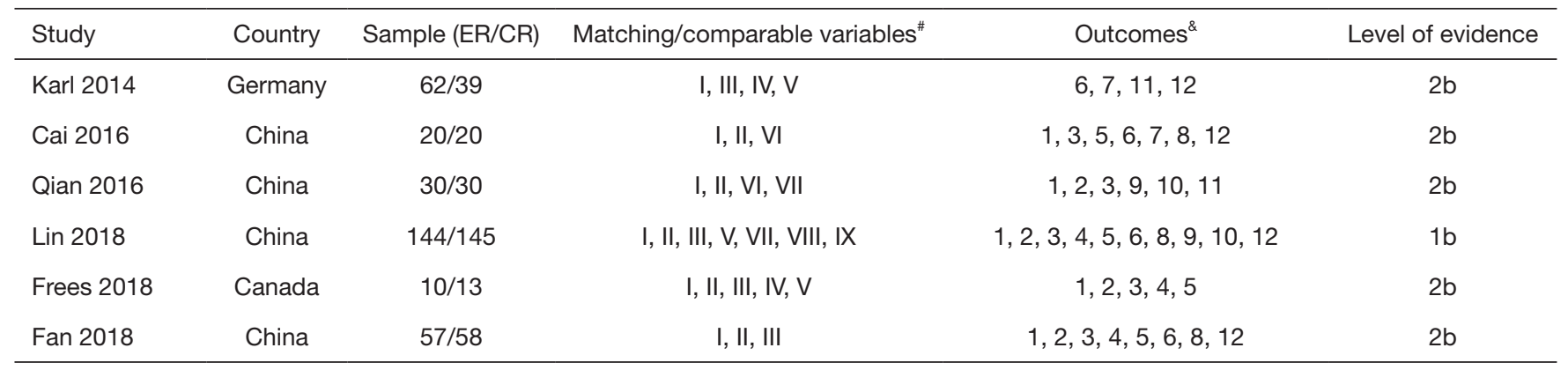

\#, I, age; II, sex; III, body mass index; IV, American Society of Anesthesiologists score; V, diversion type; VI, history of previous surgery; VII, clinical stage; VIII, concurrent CIS; IX, surgery approach. ${ }^{\&}, 1$, the time to first flatus; 2, the time to first bowel movement; 3, hospitalization time; 4, 30-day readmission; 5, overall complications; 6, ileus; 7, deep vein thrombosis; 8, infection of incision; 9, intestinal fistula; 10, leakage of urine; 11 , urinary tract infection; 12 , wound healing disorders.

0.61-2.88, $\mathrm{P}=0.48 ; \mathrm{P}=0.72, \mathrm{I}^{2}=0 \%$; Figure 3).

In general, ERAS did not significantly reduce the risk of short-term complications. In raw terms, data from 4 trials (13-16) showed that approximately $49.5 \%$ (231/467) of the ERAS patients had a complication compared with $50.5 \%(236 / 467)$ of patients in the CRAS group. Moreover, the pooled analysis did not detect significant difference between ERAS and CRAS (RR: $0.91,95 \%$ CI: $0.65-1.26, \mathrm{P}=0.56 ; \mathrm{P}=0.45, \mathrm{I}^{2}=0 \%$; Figure 3). Specifically, we did not observe significant difference in terms of ileus [4 studies $(12,13,15,16)$; RR: $0.77,95 \%$ CI: 0.50-1.18, P=0.22; Figure 4], deep vein thrombosis [2 studies $(12,15)$; RR: $0.15,95 \%$ CI: $0.02-1.20, \mathrm{P}=0.07$; Figure 4 ], infection of incision [3 studies $(13,15,16)$; RR: $0.82,95 \%$ CI: $0.40-1.66, \mathrm{P}=0.58$; Figure 4], intestinal fistula [2 studies (13,17); RR: 0.60, 95\% CI: $0.15-$ 2.48, $\mathrm{P}=0.48$; Figure 4], leakage of urine [2 studies $(13,17)$; RR: $0.63,95 \%$ CI: 0.21-1.85, P=0.40; Figure 4], and urinary tract infection [2 studies $(12,17)$; RR: $0.88,95 \%$ CI: $0.55-$ $1.39, \mathrm{P}=0.58$; Figure 4]. However, the pooled data from 4 studies $(12,13,15,16)$ showed that patients in the ERAS group had a lower risk of wound healing disorders than that in ERAS group (RR: 0.58, 95\% CI: 0.35-0.96, P=0.03; Figure 4) with no significant between-study heterogeneity $\left(\mathrm{P}=0.50, \mathrm{I}^{2}=0 \%\right)$.

\section{$R C$ with ileal conduit}

We conducted a subgroup analysis according to the type of UD. Only three studies $(13,15,17)$ containing 280 patients (141 in the ERAS group and 139 in the CRAS) were included in the final analysis for the time to first flatus. For patients undergoing RC with ileal conduit, we observed a significantly shorter time to flatus in the ERAS group compared with CRAS group (SMD: -1.43 , 95\% CI: -2.80 to $-0.07, \mathrm{P}=0.04)$. However, there was statistical heterogeneity between the trials $\left(\mathrm{P}<0.00001 ; \mathrm{I}^{2}=95 \%\right)$.

\section{Discussion}

RU with IUD is a widely used complex procedure which results in stress-induced catabolism, impaired organ function, and a reduced level of physical activity and malnutrition (13). Additionally, RC with IUD is often associated with considerable intestinal-related complications, because the ileum is often used as a substitute material for bladder in UD $(2,3,13)$. Thus, the perioperative management for patients treated with RC and IUD has been critically performed for many years. Conventionally, this procedure demand preoperative nasogastric tube insertion, preoperative intestinal preparation, and postoperative fasting, which may depress postoperative recovery (13).

ERAS pathways are standardized, multimodal, interdisciplinary protocols which involve modern anesthesia, minimally invasive techniques, optimal analgesia, and aggressive postoperative rehabilitation with early ambulation mobilization and oral nutrition $(7,13)$ and aim to improve surgical outcomes by reducing variation in perioperative best practices (7). ERAS model contained a series of perioperative treatment measures based on evidence-based medicine to reduce perioperative physical and psychological trauma and stress response, so as to accelerate the recovery process (22). For example, Yang and his colleagues (23) performed a meta-analysis involving 2 
a. The time to first flatus

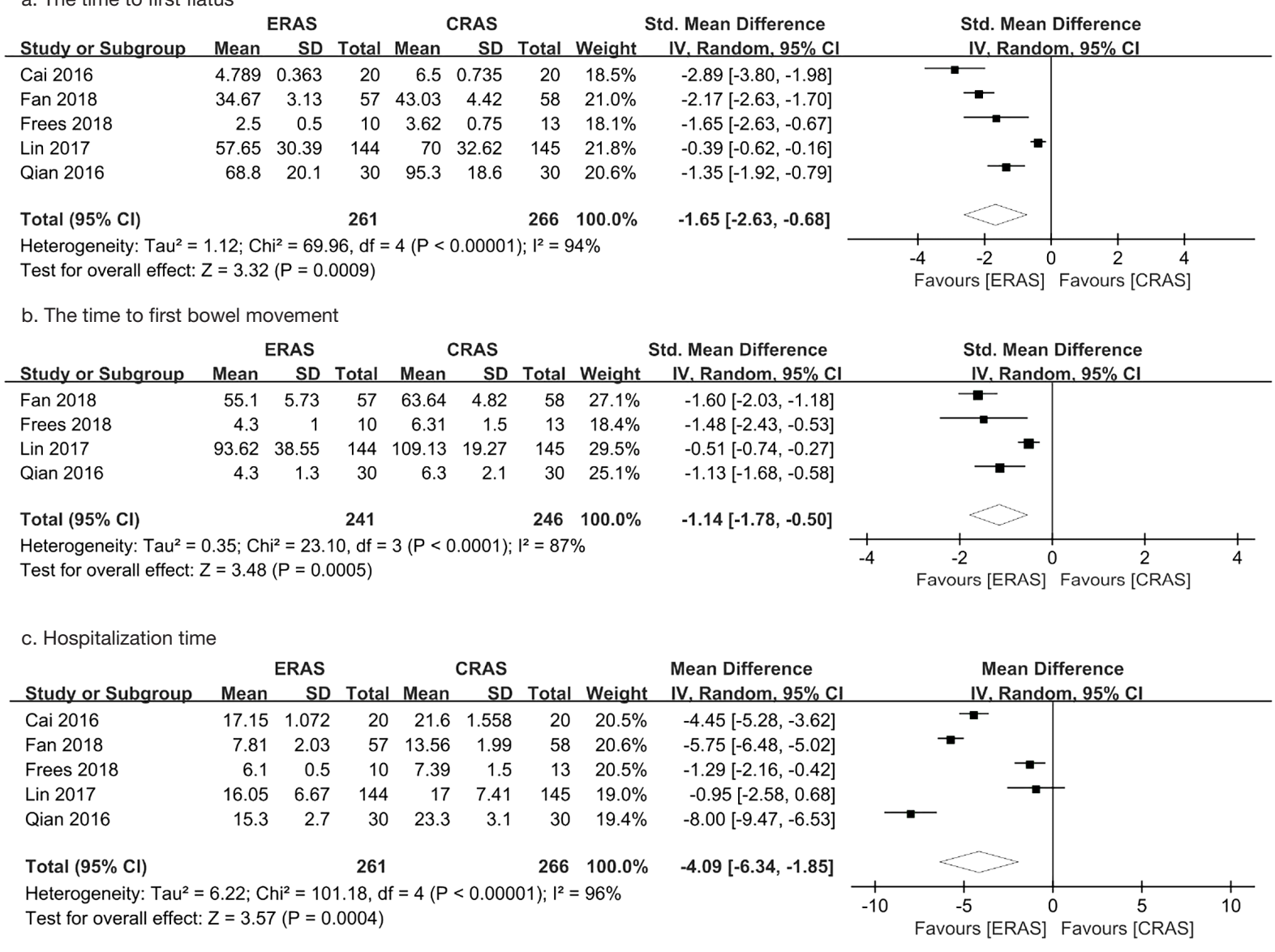

d. 30-day readmission

ERAS CRAS Risk Ratio Risk Ratio

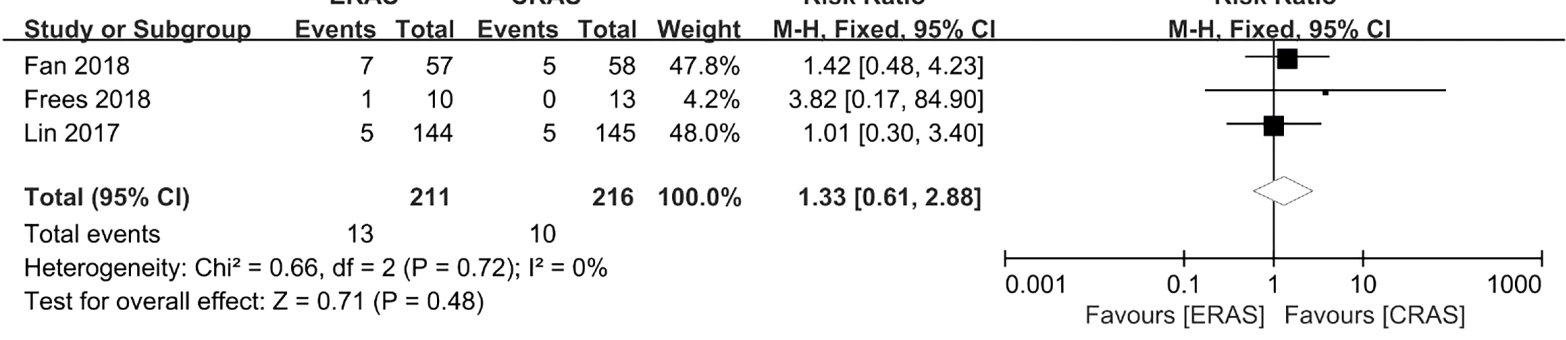

e. Overall complications

\section{ERAS CRAS}

Study or Subgroup Events Total Events Total Weight M-H, Fixed, 95\% C

Cai 2016

Fan 2018

Frees 2018

Lin 2017

Total $(95 \% \mathrm{Cl})$

Total events

Heterogeneity: $\mathrm{Chi}^{2}=2.64, \mathrm{df}=3(P=0.45) ; \mathrm{I}^{2}=0 \%$

Test for overall effect: $Z=0.58(P=0.56)$

$\begin{array}{rrrrrr}2 & 20 & 4 & 20 & 7.1 \% & 0.50[0.10,2.43] \\ 9 & 57 & 8 & 58 & 14.1 \% & 1.14[0.48,2.76] \\ 2 & 10 & 0 & 13 & 0.8 \% & 6.36[0.34,119.40] \\ 37 & 144 & 44 & 145 & 78.0 \% & 0.85[0.58,1.23] \\ & & & & & \\ & 231 & & 236 & 100.0 \% & 0.91[0.65,1.26]\end{array}$

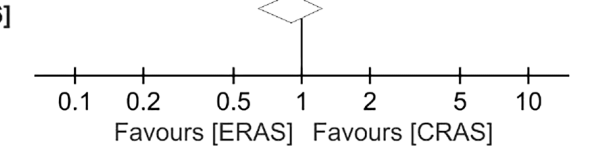

Figure 3 The outcomes of this meta-analysis. ERAS, enhanced recovery after surgery; CRAS, conventional recovery after surgery. 


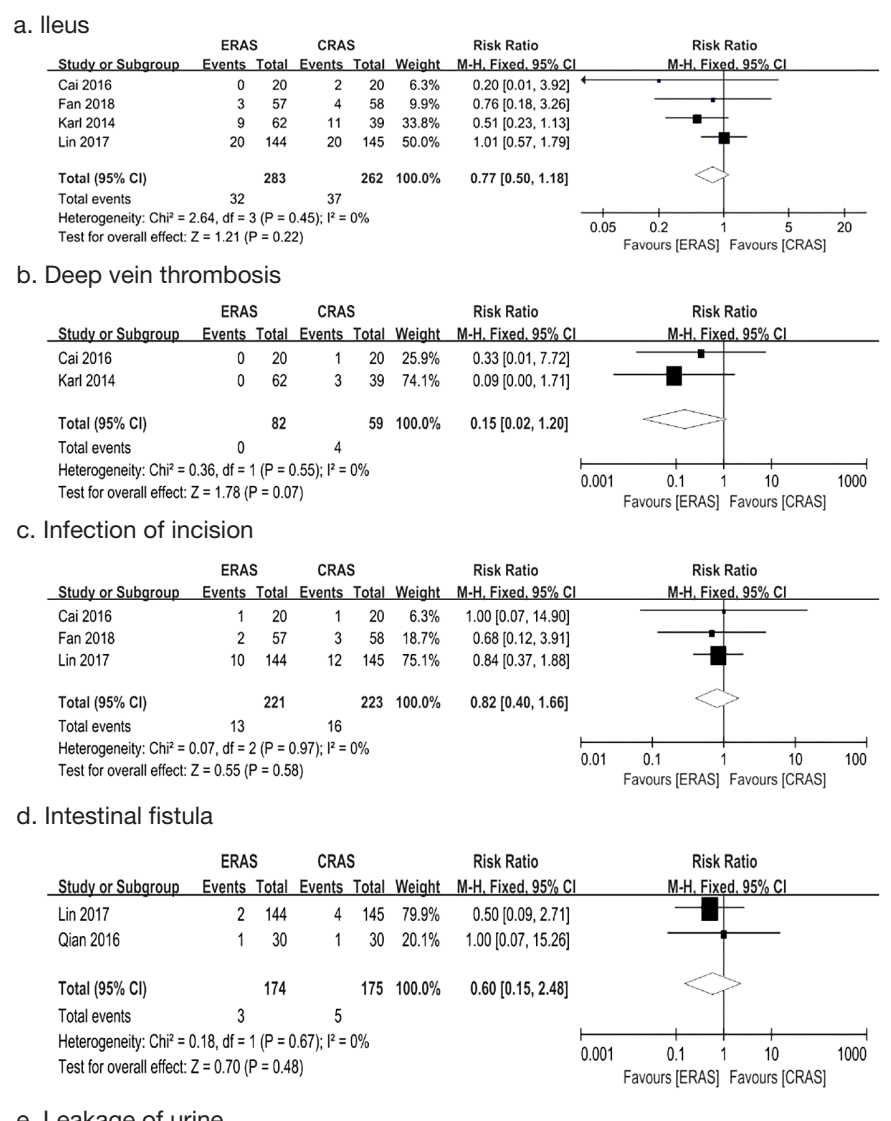

e. Leakage of urine

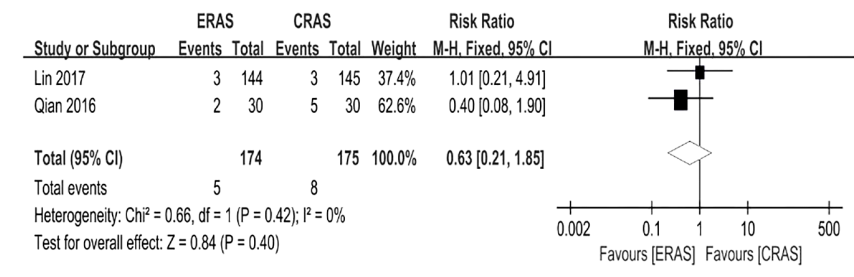

f. Urinary tract infection

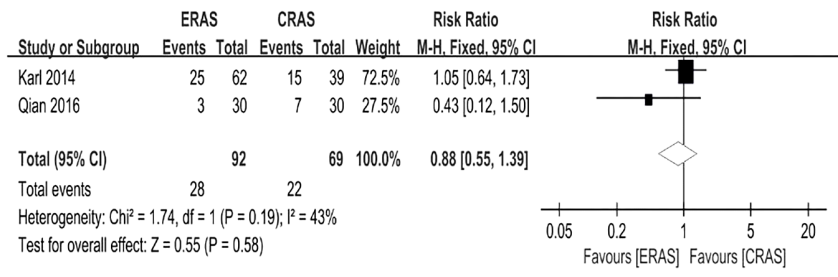

g. Wound healing disorders

\begin{tabular}{|c|c|c|c|c|c|c|c|c|c|}
\hline Study or Subgroup & $\begin{aligned} \text { ERAS } \\
\text { Events }\end{aligned}$ & & $\begin{array}{c}\text { CRAS } \\
\text { Events }\end{array}$ & & Weight & $\begin{array}{l}\text { Risk Ratio } \\
\text { M-H. Fixed, } 95 \% \mathrm{Cl}\end{array}$ & & $\begin{array}{r}\text { Risk } \\
\text { M-H. Fix }\end{array}$ & $\begin{array}{l}\text { k Ratio } \\
\text { xed. } 95 \% \mathrm{Cl}\end{array}$ \\
\hline Cai 2016 & 1 & 20 & 1 & 20 & $2.9 \%$ & $1.00[0.07,14.90]$ & & & \\
\hline Fan 2018 & 2 & 57 & 3 & 58 & $8.7 \%$ & $0.68[0.12,3.91]$ & & & \\
\hline Karl 2014 & 9 & 62 & 15 & 39 & $53.6 \%$ & $0.38[0.18,0.78]$ & & & \\
\hline Lin 2017 & 10 & 144 & 12 & 145 & $34.8 \%$ & $0.84[0.37,1.88]$ & & & \\
\hline Total $(95 \% \mathrm{Cl})$ & & 283 & & 262 & $100.0 \%$ & $0.58[0.35,0.96]$ & & & \\
\hline Total events & 22 & & 31 & & & & & & \\
\hline \multicolumn{7}{|c|}{$\begin{array}{l}\text { Heterogeneity: } \mathrm{Chi}^{2}=2.35, \mathrm{df}=3(\mathrm{P}=0.50) ; \mathrm{I}^{2}=0 \% \\
\text { Test for }\end{array}$} & 0.01 & 0.1 & $\begin{array}{lll}1 & 10 & 100\end{array}$ \\
\hline
\end{tabular}

Figure 4 The outcomes of specific complications in this meta-analysis. ERAS, enhanced recovery after surgery; CRAS, conventional recovery after surgery. 
Table 2 ERAS protocol summary of each included studies

\begin{tabular}{|c|c|c|c|c|c|c|c|c|c|c|c|}
\hline Study & \multicolumn{6}{|c|}{ Preoperative/intraoperative } & \multicolumn{4}{|c|}{ Postoperative } & Special comments \\
\hline Karl 2014 & $\mathrm{~N}$ & $\mathrm{~N}$ & NR & Y & NR & $\mathrm{N}$ & $\mathrm{Y}$ & $\mathrm{Y} / \mathrm{Y}$ & $\mathrm{Y}$ & 24 to $48 \mathrm{~h}$ & - \\
\hline Cai 2016 & $\mathrm{Y}$ & $\mathrm{Y}$ & NR & NR & NR & $\mathrm{N}$ & NR & $Y / Y$ & $\mathrm{Y}$ & NR & - \\
\hline $\begin{array}{l}\text { Qian } \\
2016\end{array}$ & $\mathrm{Y}$ & $\mathrm{Y}$ & $\mathrm{Y}$ & NR & NR & $\mathrm{N}$ & NR & $Y / Y$ & NR & NR & $\begin{array}{c}\text { Prolonging oxygen inhalation after } \\
\text { surgery }\end{array}$ \\
\hline Fan 2018 & $\mathrm{Y}$ & $\mathrm{Y}$ & NR & $\mathrm{Y}$ & $\mathrm{Y}$ & $\mathrm{N}$ & NR & $Y / Y$ & $\mathrm{Y}$ & NR & Low molecular weight heparin \\
\hline $\begin{array}{l}\text { Frees } \\
2018\end{array}$ & $\mathrm{Y}$ & $\mathrm{N}$ & $\mathrm{Y}$ & $\mathrm{Y}$ & $\mathrm{Y}$ & NR & $\mathrm{Y}$ & $\mathrm{Y} / \mathrm{Y}$ & NR & NR & $\begin{array}{l}\text { Low molecular weight heparin, } \\
\text { compression stockings, chewing gum }\end{array}$ \\
\hline
\end{tabular}

${ }^{a}$, if the NGT was removed at the end of the surgery, the study was classified as not leaving an NGT postoperatively; ${ }^{b}$, preoperative education includes operation related risks, individualized psychological care and specific rapid rehabilitation measures. CL, carbohydrate loading; EDA, epidural anesthesia; EM, early mobilization; EOF, early oral feeding; FM, goal-directed fluid management; MBP, mechanical bowel preparation; POE, preoperative education; N, no; NGT, nasogastric tube; NR, not reported; Y, yes.

RCTs and 2 cohort studies and they showed that the use of comprehensive bowel preparation for UD surgery using ileum does not offer any significant advantage over limited bowel preparation. A meta-analysis conducted by Zhao et al. (24) showed that the insertion of nasogastric tube after $\mathrm{RC}$ with UD cannot shorten the time of gastrointestinal function recovery or reduce the incidence of complications. Additionally, the previous review and meta-analysis showed that preoperative carbohydrate loading or hydration (25), goal-directed therapy $(26,27)$, chewing gum (28) and prokinetics (29) showed advantages of gastrointestinal recovery after RC. Furthermore, Deibert et al. (30) conducted the first RCT to evaluate the impact of early feeding on recovery after RC and they indicated that ERAS protocols standardize postoperative care and early feeding is a well-tolerated addition. Table 2 details the ERAS protocols of included studies. Only 2 studies $(12,14)$ have no preoperative mechanical bowel preparation, and almost all studies $(12,13,15,17)$ have no preoperative nasogastric tube. Besides, all studies require postoperative early mobilization and early oral feeding. Notably, attention should also be paid to preoperative education (13-17).

Urologists began to pay attention to the application of ERAS in urological surgery due to the great success of ERAS in the postoperative management of other surgeries, especially in colorectal surgery $(12,31,32)$. Pruthi and his colleague (33) conducted a retrospective study in 2003 and they found ERAS could reduce morbidity and improve recovery with regard to the early institution of an oral diet and early hospital discharge. Since then, many observational studies and a few RCTs have been conducted and four meta-analysis (6-9) have been performed attempting to determining the efficacy of ERAS for the management of RC with UD. Most of the studies included in these metaanalysis (6-9) were observational studies and their findings supported the implementation of ERAS with regard to length of stay and the time to recovery of bowel function. However, complications are controversial. Several studies $(6,9)$ concluded that ERAS group had a lower rate of overall complications than that of CRAS group, whereas others did not $(7,8)$. Only one study (6) summarized specific complications and the findings indicated that postoperative paralytic ileus was significantly lower in the ERAS group than that in the CRAS group. Furthermore, the UD modalities included in these meta-analyses (6-9) were inconsistent. Thus, we think it is necessary to conduct this systematic review and meta-analysis of RCTs comparing ERAS to CRAS to confirm the clinical efficacy and safety of ERAS protocols.

Similar to the previous studies (6-9), our study showed that ERAS protocols might accelerate gastrointestinal recovery and shorten length of stay as a result of shorter time to first flatus and first bowel movement, and shorter hospitalization time. Additionally, we also observed no significant difference with regard to 30-day readmission. Unlike the previous meta-analysis (6), our meta-analysis showed no significant difference between ERAS group and CRAS group in terms of postoperatively overall complications and postoperative ileus. However, we found that patients in the ERAS group had a lower risk of wound 
healing disorders than that in ERAS group. We believe that the focus of ERAS program is on management of postoperative rehabilitation. Early recovery of intestinal function can promote early regular diet, thereby creating conditions for wound healing. Other complications may be mainly determined by the operation itself. Therefore, shorter time to flatus and bowel movement are unlikely to improve the overall complication rate. In addition, we think that early recovery of gastrointestinal function might facilitate wound healing and early mobilization, thereby reducing the incidence of basic complications such as cardiopulmonary disease. However, due to the limitation of available data, we cannot assess the time to first mobilization and the risk of basic diseases such as cardiovascular disorders. Moreover, early functional rehabilitation may improve patients' psychological trauma and stress response, increase self-confidence in treatment and motivation to cooperate with treatment, and then contribute to unexpected benefits.

Our study did have the following limitations. Firstly, the heterogeneity of this study is mainly derived from the specific measures of ERAS protocol between the trials, and the other heterogeneity includes the surgical approaches [one study with open radical cystectomy (ORC), laparoscopic radical cystectomy (LRC) and robot-assisted radical cystectomy (RARC) (13), three studies with LRC $(15-17)$, and two studies $(12,14)$ with no specification], the study population, the experience of the surgeon, and the definition of the outcomes measures. Secondly, our previous meta-analysis indicated that minimally invasive approaches could be considered as a feasible and safe alternative to ORC when performed by experienced surgeons in selected patients (34), and patients with RARC may benefit from significantly lower complications, shorter LOS, higher lymph node yield and lower death risk (35). In this study, we are unlikely to assess the impact of surgical approaches on EARS due to the limitation of available data. Thirdly, emphasis of ERAS protocol is placed on the management of rehabilitation after RC. However, we are unable to evaluate early mobilization and the risk of basic diseases such as cardiovascular disorders. Last, the limited RCTs and the rather small sample make it difficult to emphatically confirm the advantages of ERAS. Further large prospective randomized, double-blind study conducted by large volume and experienced surgeons are warranted to confirm our findings and definitively resolve this important controversy in urology. Besides, high-quality meta-analysis based on high-quality RCTs is still needed to improve the elements of ERAS protocols.

Our research has initially confirmed the efficacy and safety of ERAS protocol on management of patients undergoing. The direction and focus of future researches are to achieve a comprehensive and standardized approach throughout the perioperative period based on evidencebased medicine.

\section{Conclusions}

Our findings indicated that ERAS protocols throughout the perioperative period of RC with IUD might reduce hospitalization expenses and contribute to higher turnover ward, more efficient utilization of medical resources and lower risk of nosocomial infection as a result of shorter length of stay. Besides, early rehabilitation of gastrointestinal function might not only facilitate wound healing and early mobilization, thereby reducing the incidence of basic complications such as cardiopulmonary disease, but also improve patients' psychological trauma and stress response, increase self-confidence and motivation in treatments, and then lead to unexpected benefits. Further large volume, multicenter randomized controlled studies are warranted before making the final clinical guidelines.

\section{Acknowledgments}

Funding: This work was supported by the 1.3 .5 project for disciplines of excellence, West China Hospital, Sichuan University (ZY2016104), Pillar Program from Department of Science and Technology of Sichuan Province (2018SZ0219) and 1.3.5 Project for Disciplines of Excellence-Clinical Research Incubation Project, West China Hospital, Sichuan University (2018HXFH049). The funders had no role in study design, data collection or analysis, preparation of the manuscript, or the decision to publish.

\section{Footnote}

Reporting Checklist: The authors have completed the PRISMA reporting checklist. Available at http://dx.doi. org/10.21037/tau-19-941

Conflicts of Interest: All authors have completed the ICMJE uniform disclosure form (available at http://dx.doi. org/10.21037/tau-19-941). The authors have no conflicts of interest to declare. 
Ethical Statement: The authors are accountable for all aspects of the work in ensuring that questions related to the accuracy or integrity of any part of the work are appropriately investigated and resolved.

Open Access Statement: This is an Open Access article distributed in accordance with the Creative Commons Attribution-NonCommercial-NoDerivs 4.0 International License (CC BY-NC-ND 4.0), which permits the noncommercial replication and distribution of the article with the strict proviso that no changes or edits are made and the original work is properly cited (including links to both the formal publication through the relevant DOI and the license). See: https://creativecommons.org/licenses/by-nc-nd/4.0/.

\section{References}

1. Bray F, Ferlay J, Soerjomataram I, et al. Global cancer statistics 2018: GLOBOCAN estimates of incidence and mortality worldwide for 36 cancers in 185 countries. CA Cancer J Clin 2018;68:394-424.

2. Witjes JA, Bruins HM, Cathomas R, et al. Guidelines on muscle-invasive and metastatic bladder cancer. The Netherlands: European Association of Urology. Access date 2019. Available online: https://uroweb.org/guideline/ bladder-cancer-muscle-invasive-and-metastatic/\#7

3. Su S, Gu L, Ma X, et al. Comparison of Laparoscopic and Robot-assisted Radical Cystectomy for Bladder Cancer: Perioperative and Oncologic Outcomes. Clin Genitourin Cancer 2019;17:e1048-53.

4. Kehlet H. Multimodal approach to control postoperative pathophysiology and rehabilitation. Br J Anaesth 1997;78:606-17.

5. Nicholson A, Lowe MC, Parker J, et al. Systematic review and meta-analysis of enhanced recovery programmes in surgical patients. Br J Surg 2014;101:172-88.

6. Xiao J, Wang M, He W, et al. Does Postoperative Rehabilitation for Radical Cystectomy Call for Enhanced Recovery after Surgery? A Systematic Review and Metaanalysis. Curr Med Sci 2019;39:99-110.

7. Tyson MD, Chang SS. Enhanced Recovery Pathways Versus Standard Care After Cystectomy: A Meta-analysis of the Effect on Perioperative Outcomes. Eur Urol 2016;70:995-1003.

8. Giannarini G, Crestani, A, Inferrera A, et al. Impact of enhanced recovery after surgery protocols versus standard of care on perioperative outcomes of radical cystectomy: a systematic review and meta-analysis of comparative studies. Minerva Urol Nefrol 2019;71:309-23.

9. Cheng H, Si S, Zheng Y, et al. Enhanced Recovery Pathways versus Standard Care after Cystectomy: A Metaanalysis and Systemtic Review. Journal of International Translational Medicine 2018;6:25-33.

10. Moher D, Liberati A, Tetzlaff J, et al. Preferred reporting items for systematic reviews and meta-analyses: the PRISMA statement. PLoS Med 2009;6:e1000097.

11. Centre for Evidence-Based Medicine. Oxford Centre for evidence-based medicine: levels of evidence, 2009. Available online: http://www.cebm.net/oxfordcentreevidence-based-medicine-levels-evidencemarch-2009/

12. Karl A, Buchner A, Becker A, et al. A new concept for early recovery after surgery for patients undergoing radical cystectomy for bladder cancer: results of a prospective randomized study. J Urol 2014;191:335-40.

13. Lin T, Li K, Liu H, et al. Enhanced recovery after surgery for radical cystectomy with ileal urinary diversion: a multi-institutional, randomized, controlled trial from the Chinese bladder cancer consortium. World J Urol 2018;36:41-50.

14. Frees SK, Aning J, Black P, et al. A prospective randomized pilot study evaluating an ERAS protocol versus a standard protocol for patients treated with radical cystectomy and urinary diversion for bladder cancer. World J Urol 2018;36:215-20.

15. Cai $Z$, Peng $Y$, Huang $X$, et al. Fast track surgery nursing in the perioperative management of laparoscopic radical cystectomy. Medical Journal of West China 2016;28:1168-71.

16. Fan J, Zhang H, He W, et al. Application of enhanced recovery after surgery in perioperative period of radical cystectomy and evaluation on its effect. Journal of Chongqing Medical University 2018;43:552-5.

17. Qian P, Gao X, Liu B, et al. Nursing of fast track surgery for patients with radical cystectomy and ileal conduit in laparoscopic perioperative period. International Journal of Urology and Nephrology 2016;36:204-6.

18. Hozo SP, Djulbegovic B, Hozo I. Estimating the mean and variance from the median, range, and the size of a sample. BMC Med Res Methodol 2005;5:13.

19. Yang L, Wang G, Du Y, et al. Remote ischemic preconditioning reduces cardiac troponin I release in cardiac surgery: a meta-analysis. J Cardiothorac Vasc Anesth 2014;28:682-9.

20. Higgins JP, Thompson SG, Deeks JJ, et al. Measuring inconsistency in meta-analyses. BMJ 2003;327:557-60. 
21. Higgins JP, Thompson SG. Quantifying heterogeneity in a meta-analysis. Stat Med 2002;21:1539-58.

22. Kehlet H, Wilmore DW. Multimodal strategies to improve surgical outcome. Am J Surg 2002;183:630-41.

23. Yang L, Chen HS, Welk B, et al. Does using comprehensive preoperative bowel preparation offer any advantage for urinary diversion using ileum? A metaanalysis. Int Urol Nephrol 2013;45:25-31.

24. Zhao T, Huang L, Tian Y, et al. Is it necessary to insert nasogastric tube routinely after radical cystectomy with urinary diversion? A meta-analysis. Int J Clin Exp Med 2014;7:4627-34.

25. Pre-Operative Carbohydrate Loading or Hydration: A Review of Clinical and Cost-Effectiveness, and Guidelines. Ottawa ON: 2016 Canadian Agency for Drugs and Technologies in Health, 2016.

26. Giglio MT, Marucci M, Testini M, et al. Goal-directed haemodynamic therapy and gastrointestinal complications in major surgery: a meta-analysis of randomized controlled trials. Br J Anaesth 2009;103:637-46.

27. Gómez-Izquierdo JC, Feldman LS, Carli F, et al. Meta-analysis of the effect of goal-directed therapy on bowel function after abdominal surgery. Br J Surg 2015;102:577-89.

28. Su'a BU, Pollock TT, Lemanu DP, et al. Chewing gum and postoperative ileus in adults: a systematic literature review and meta-analysis. Int J Surg 2015;14:49-55.

Cite this article as: Feng D, Liu S, Lu Y, Wei W, Han P. Clinical efficacy and safety of enhanced recovery after surgery for patients treated with radical cystectomy and ileal urinary diversion: a systematic review and meta-analysis of randomized controlled trials. Transl Androl Urol 2020;9(4):1743-1753. doi: 10.21037/tau-19-941
29. Cui Y, Chen H, Qi L, et al. Effect of alvimopan on accelerates gastrointestinal recovery after radical cystectomy: A systematic review and meta-analysis. Int J Surg 2016;25:1-6.

30. Deibert CM, Silva MV, RoyChoudhury A, et al. A Prospective Randomized Trial of the Effects of Early Enteral Feeding After Radical Cystectomy. Urology 2016;96:69-73.

31. Kehlet H. Fast-track colorectal surgery. Lancet 2008;371:791.

32. Khoo CK, Vickery CJ, Forsyth $\mathrm{N}$ et al. A prospective randomized controlled trial of multimodal perioperative management protocol in patients undergoing elective colorectal resection for cancer. Ann Surg 2007;245:867.

33. Pruthi RS, Chun J, Richman M. Reducing time to oral diet and hospital discharge in patients undergoing radical cystectomy using a perioperative care plan. Urology 2003;62:661-5; discussion 665-6.

34. Feng D, Li A, Hu X, et al. Comparative effectiveness of open, laparoscopic and robot-assisted radical cystectomy for bladder cancer: a systematic review and network metaanalysis. Minerva Urol Nefrol 2020;72:251-64.

35. Feng D, Liu S, Tang Y, et al. Comparison of perioperative and oncologic outcomes between robot-assisted and laparoscopic radical cystectomy for bladder cancer: a systematic review and updated meta-analysis. Int Urol Nephrol 2020;52:1243-54. 